\title{
CHRONICLE AND INFORMATION
}

\section{RECOOP HST Consortium}

Members of the RECOOP HST Consortium realized that without high quality science there is no innovation!

The main activity of the RECOOP HST Consortium is research networking to build multinational and multidisciplinary research teams to be able to investigate complex diseases that are the major public health problems (cardiovascular, cancer, neurodegenerative diseases, women's health and human development) in Central and Eastern Europe (CEE) and worldwide. The members of RECOOP HST Consortium decided to review the scientific progress in the Consortium and therefore started «Bridges in Life Sciences» meetings.

The first «Bridges in Life Sciences» meeting was organized by Cedars-Sinai Medical Center (CSMC) in collaboration with Fogarty International Center, National Institute of Health USA (NIH), and it was held in Budapest, Hungary in October 2003. That meeting highlighted US-Central and Eastern European research collaborations in eight scientific tracks, providing an excellent networking opportunity for NIH-supported scientists from over ten CEE countries. The eight priority areas were: cancer, reproductive health, cardiovascular health, infectious diseases, neurosciences, functional genomics/proteomics and cell biology, environmental health, and behavioral health studies.

The second «Bridges in Life Sciences» Annual Scientific Meetings was on October 5, 2007 in Pecs, Hungary and the third in Zagreb, Croatia on October 4, 2008. The «Bridges in Life Sciences» $4^{\text {th }}$ Annual Scientific Meeting took place in Debrecen, Hungary on April 4-5, 2009. In Debrecen the RECOOP Consortium provided opportunities for young scientists to discuss the major public health problems in the region and engaged with senior scientists from RECOOP, Visegrad Group

(C) Institute of Molecular Biology and Genetics NAS of Ukraine, 2010
(V4) academic organizations and NIH Institutes funded PIs from the USA with robust collaborative works in the CEE countries.

The «Bridges in Life Sciences» $5^{\text {th }}$ Annual Scientific Meetingwas organized for the RECOOP HST member organizations and also for Universities and Research Institutes in Lviv, Ukraine on April 9-11, 2010.

Since 2003, CSMC has implemented a systemic training program to support partner organizations in CEE as they build their capacity in the field of research and innovation management. In 2006, CSMC, with eleven CEE universities from six countries (Croatia, Czech Republic, Hungary, Romania, Slovakia, and Ukraine), formed the Regional Cooperation for Health, Science and Technology (RECOOP HST) Consortium:

Cedars-Sinai Medical Center, Los Angeles, California; Faculty of Military Health Sciences, University of Defense, Hradec Kralove, Czech Republic;

School of Medicine University J. J. Strossmayer Osijek, Croatia;

University of Debrecen, Hungary;

University of Pecs, Hungary;

University of Szeged, Hungary;

Carol Davila University of Medicine and Pharmacy, Bucharest, Romania;

Slovak Medical University, Bratislava, Slovakia;

Institute of Molecular Biology and Genetics, National Academy of Science of Ukraine, Kyiv, Ukraine;

Palladin Institute of Biochemistry, National Academy of Sciences of Ukraine, Kyiv, Ukraine;

Institute of Cell Biology, National Academy of Sciences of Ukraine, Lviv, Ukraine;

Danylo Halytsky Lviv National Medical University, Lviv, Ukraine.

The RECOOP HST Consortium is building an inventory of research tools (cell lines, test kits, antibodies, markers), which would be used to expand the 
research output of each participating organization. The Consortium also is enhancing the institutions' Research and Development success rates by licensing Intellectual Property (IP) to industry via the development of a joint patent portfolio and creation of a Patent Warehouse. In addition, the Consortium has created a Joint Clinical Trial Management (JCTM) Team and is building Multidisciplinary Research Networks to enhance the competitiveness of the participating academic organizations in attracting sponsored research. In 2006 and 2007, the Consortium organized Research and Innovation Management Summer Schools, utilizing distance learning to provide training IP for Ph. D. students as part of their credit requirement towards completion of their doctoral thesis.

The main goal of the RECOOP HST Consortium is to enhance research collaboration in CEE (EU-V4) and to support and provide a platform for scientific networking in the life sciences within the all-European integration. CSMC and the RECOOP HST Consortium aim at promoting the development of closer collaboration with the CEE and the Visegrad Four (V4) countries - Czech Republic, Hungary, Poland, and Slovakia (http://www.visegradgroup.ed) - by supporting common scientific and educational projects, regio- nal cooperation in research and development and the Visegrad Scholarship Program. The CSMC and RECOOP HST Consortium are planning to provide matching fund for each successful Visegrad Scholarship Application. The hosts Universities will receive 2,500 Euro to help them in the implementation of a successful research program with the visiting young scholar (Ph. D. student or post doc).

"The RECOOP HST Consortium is exploring and enhancing the LOCAL scientific outputs of the partner organizations, creating a critical mass of scientifically sound innovative biotechnologies at the REGIONAL level and exploiting the outcomes on the GLOBAL market.» ${ }^{T M}$
Sandor G. Vari, MD

Director, International Research and Innovation Management Program Cedars-Sinai Medical Center \&

General Manager of the RECOOP HST Consortium \&

member of Editorial Board of Biopolymers and cell 